\begin{tabular}{c} 
International Journal of Engineering \& Technology, $7(2.33)(2018) 346-349$ \\
International Journal of Engineering \& Technology \\
SPC \\
Website: $w$ ww.sciencepubco.com/index.php/IJET \\
Research paper \\
\hline
\end{tabular}

\title{
Usefulness of tungsten shield in mammography
}

\author{
Chang-Gyu Kim * \\ Department of Radiological Science, Gimcheon University, Gimcheon City, Gyung- buk, 39528, South Korea \\ *Corresponding author E-mail: radkcg@hanmail.net
}

\begin{abstract}
Background/Objectives: In order to minimize unnecessary artifacts and medical radiation dose for acquiring final diagnostic medical image with high diagnostic value in X-ray mammography, a tungsten shield was produced to evaluate the usefulness of clinical application.

Methods/Statistical analysis: The radiation dose at the time of mammography was measured in the same posture as breast cancer examination in a clinical hospital for health screening. In the R - CC, L - CC. R - MLO, L- MLO posture mammography, the distribution of radiation dose for each part by placing a glass dose device at the breast position with the breast equivalent phantom. In order to reduce measurement error, mammography was repeated five times.

Findings: When the eco-friendly shield was used, the average dose was $40 \pm 0.34 \mu \mathrm{Gy}$, which showed a radiation dose reduction efficiency of $64.6 \%$ and a reduction factor of 2.8 . Using eco-friendly shield tungsten shield, reproducibility of fibrin was 4.5 , reproducibility of specks was 4.0, and reproducibility of the mass was $4.0 \pm 0.2$, resulting in 12.5, exceeding the approved acceptance criteria of 10 .

Improvements/Applications: This result is expected to be used as an important basic data for predicting patient's radiation dose, determining test method and decreasing medical radiation dose in future breast cancer examination.
\end{abstract}

Keywords: Mammography; Shield; Tungsten; Usefulness; Glass Dosimeter.

\section{Introduction}

Early diagnosis and accurate diagnosis of breast cancer is very important to increase the probability of breast cancer treatment. Mammography, breast ultrasonography, tissue examination with excisional biopsy, and magnetic resonance imaging (MRI) have been used for the diagnosis of breast cancer [1], [2].

In the case of tissue examination with excisional biopsy resecting only a part of the lesion, a follow-up examination of the remaining lesions is required, scarring and insertion of repeated syringe needles have been suggested as inconveniences of examination, breast ultrasound is known to be difficult to distinguish between malignant and benign tumors, and it is known that it is difficult to detect when the size of breast cancer is small [3], [4].

MRI-based breast cancer examination is known to be more accurate than mammography or ultrasound to assess the size of breast cancer, but its utilization is relatively low due to the cost of examination [5]. Digital mammography is the most excellent and widely used method for diagnosis of micro calcification lesions and cancer, other than diagnosing lesions by touch [6].Because the breast region is known to be more sensitive to radiation than other regions of the human body, digital mammography using $\mathrm{X}$-rays has been on the rise for the purpose of early detection of breast cancer [7].

Mammography is characterized by small X-ray absorptions of lesion and surrounding tissues such as mammary gland tissue and adipose tissue, and the need to reveal fine calcifications. In order to improve the detection rate of breast lesions, a small focus is used and the use of a small focus results in an increase of the exposure time to increase the radiation dose [4], [7] It has been reported that the amount of radiation the patient receives during breast examination may cause more radiation exposure than the benefit of early detection [8].

The glass dosimeter is capable of repeated measurement and readout of the radiation radiation dose because the silver ion particle fluorescence center produced by irradiation is not destroyed by repeated measurement. In addition, the measurable dose range is $10 \mu \mathrm{Gy}$ to $500 \mathrm{~Gy}$, and it has wide measurement range, low fading effects and excellent direction and energy dependence [9-13].

Tungsten is an off-white solid metal with an atomic number of 74, which has the meaning of 'heavy stone'. It is heavy and hard, has the highest melting point of $3,422^{\circ} \mathrm{C}$ among metal elements, and has the lowest vapor pressure, making it widely used as an incandescent filament and various electrical and electronic materials. Recently, tungsten shield has been attracting attention as an ecofriendly X-ray shielding defense due to the hazard to human body of lead material [14-16].

Studies involving the reduction of breast radiation dose include, evaluation of radiation dose by exposure method and program treatment [7], study on absorption dose and lead shielding in breast tissue using simulation [17], [18], study on image evaluation using Monte Carlo simulation [19], and evaluation of the permeation dose considering the compression pad material of the mammography equipment [20]. However, these studies have been based on dose assessment by apparatus or accessories, and there is no study of radiation dose using eco-friendly shield and glass dosimeter.

This study was performed to produce an eco-friendly tungsten shield in mammography with high radiation tissue weighting factor and to measure radiation dose using glass dosimeter to provide basic data to minimize radiation dose and acquire optimal images. 


\section{Subjects and methods}

\subsection{Materials and equipment}

The radiation generator was a Phillips LORAD selonia system [Figure1]. To measure the radiation dose of the subject, a full body phantom (Model PBU-31, Kyoto Kagaku, Japan) consisting of human body equivalent material and a glass dosimeter Dose Ace (Model GD-352M and FGD-1000, Asahi Techno Glass Cooperation, Shizuoka, Japan) were used [Figure2].

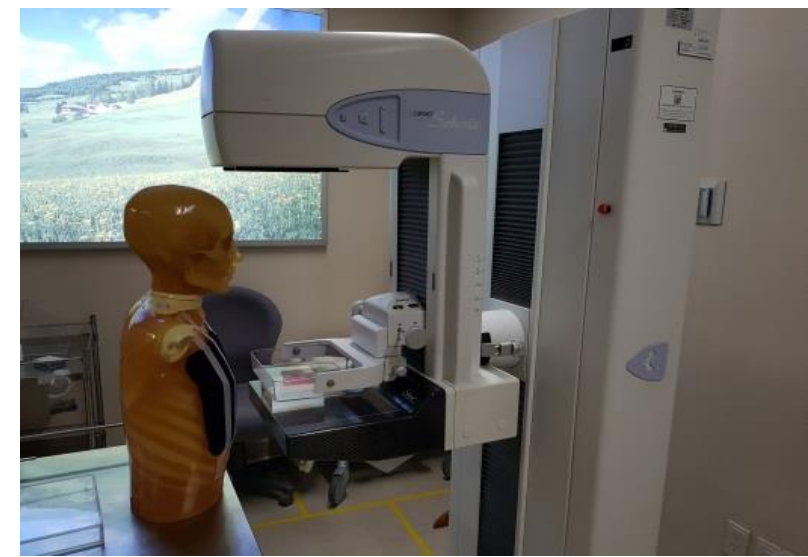

Fig. 1: Radiographic Equipment and Full Body Phantom.

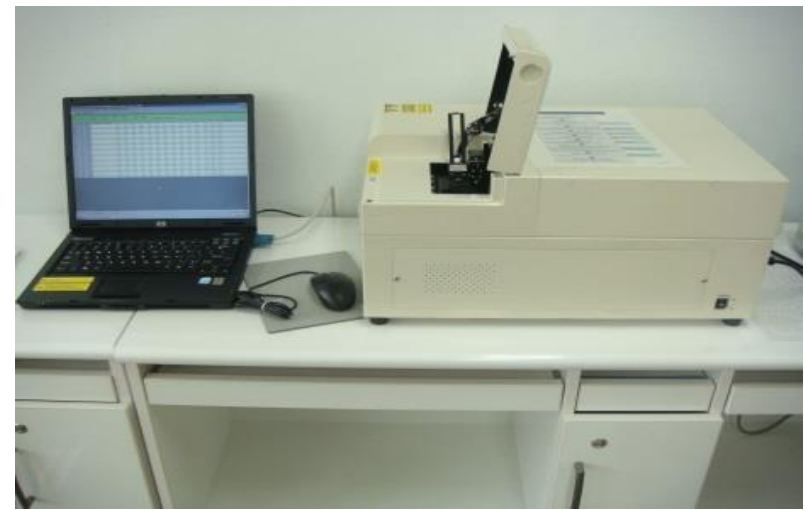

Fig. 2: Glass Dosimeter.

\subsection{Measurement of radiation dose with or without eco- friendly shield during mammography}

The radiation dose at the time of mammography was measured in the same posture as breast cancer examination in a clinical hospital for health screening. In the R - CC, L - CC. R - MLO, L- MLO posture mammography, the distribution of radiation dose for each part by placing a glass dose device at the breast position with the breast equivalent phantom. In order to reduce measurement error, mammography was repeated five times [Figure3].

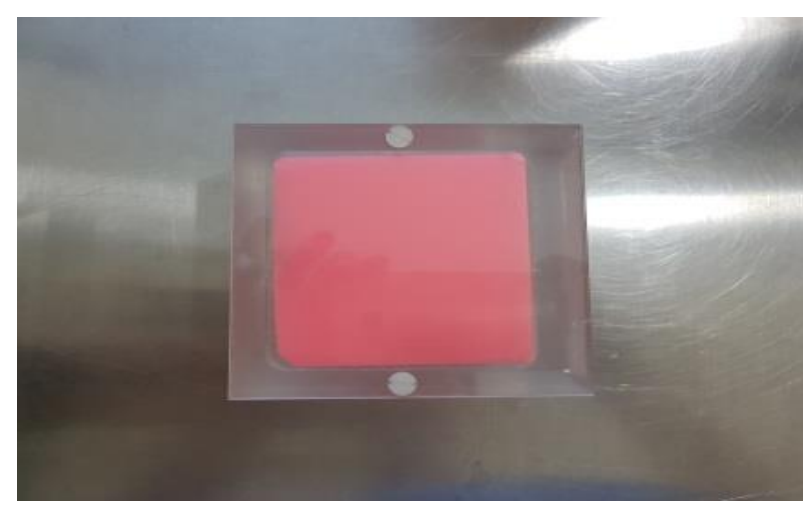

Fig. 3: Breast Equivalent Material Phantom.

\subsection{Glass dosimeter dose measurement}

The calibration of the glass dosimeter was performed using a glass element irradiated with $6 \mathrm{mGy}$ using a ${ }^{137} \mathrm{Cs}$ standard source at the Japan Radiation Standards Institute. In consideration of the characteristics of the element, the annealing process was performed at $400^{\circ} \mathrm{C}$ for 1 hour before the irradiation, and the background was measured as $10-20 \mu \mathrm{Gy}$ after cooling, and after panoramic scan, the pre-heating was performed at $70^{\circ} \mathrm{C}$ for 1 hour, then cooled, and the dose value irradiated to the element was measured by a reader where dose accumulated value was repeated 10 times and mean value and standard deviation were calculated. The radiation dose value was derived by subtracting the background value from the calculated value [9 13].

\subsection{Image evaluation method}

Qualitative analysis of breast tissue equivalent phantom was performed to evaluate the image quality of images with or without eco-friendly shield. Qualitative analysis consisted of evaluating the number of fibers, specks, and masses reproduced in the image by one radiology specialist and five radiologists with more than 10years experience. The standard of number of fibers was 6 , and the number of specks and masses was 5 .

\subsection{Statistical processing and analysis}

Data were analyzed using the SPSSWIN (Ver 13.0) statistical program, and t-test and ANOVA were performed to verify the significance of the mean values of the radiation dose measurements in the control and experimental groups. The significance level of all statistics was $\mathrm{p}<0.05$.

\section{Results and discussion}

\subsection{Dose reduction effect of eco-friendly shield in mammography examination}

Medical exposure refers to the exposure of a patient or an individual to radiation for medical treatment at a medical facility, which is distinct from the occupational exposure of a medical worker [21].Radiation dose measurements during breast imaging were measured in postures for a clinical hospital setting for breast cancer examination for health screening purposes. In the R - CC, L CC, R - MLO, L- MLO posture mammography, the distribution of radiation dose for each part by placing a glass dose device at the breast position with the breast equivalent phantom.

For R - CC posture, $29 \mathrm{kVp} 72.2 \mathrm{mAs}$, Mo filter was used, for L CC posture, $29 \mathrm{kVp} 76.9 \mathrm{mAs}$, Mo filter was used, for R - MLO posture, $28 \mathrm{kVp} 83.5 \mathrm{mAs}$, Mo filter was used, and for L- MLO posture, $28 \mathrm{kVp} 77.8 \mathrm{mAs}$, Mo filter was used. In order to reduce measurement error, mammography was repeated 5 times.

In glass dosimeters used in radiation dose measurement, electrons and holes PO4 are generated when silver ion activated phosphate of glass element is irradiated with ionizing radiation, the generated electrons are captured by $\mathrm{Ag}+$ in the glass element to become Ago, and the holes are trapped in $\mathrm{PO} 4+$, or migrate to $\mathrm{Ag}+$ over time to form $\mathrm{Ag}++$. These $\mathrm{Ago}$ and $\mathrm{Ag}++$ form a more stable state RPL (Radio Photo Luminescence) center. When irradiated with ultraviolet rays at the center of RPL, energy is absorbed to create excited state, and then it returns to the stable state and emits orange fluorescence. At this time, the number of RPL centers emitting fluorescence is constant and can be read out repeatedly since it does not disappear in the surrounding environment and reading process. This feature is the biggest difference when compared to a thermo luminescence dosimeter (TLD) that can only read once. Also, the amount of fluorescence emitted from the glass dosimeter is proportional to the amount of irradiated radiation, and the dose accumulated through simple heat treatment at $400^{\circ} \mathrm{C}$ for one hour is reset and can be reused [22]. 
For the results of the radiation dose measurement according to the use of eco-friendly shield in mammography, the mean dose was $113 \pm 1.06 \mu \mathrm{Gy}$ when no shield was used and the mean dose was $40 \pm 0.34 \mu \mathrm{Gy}$ when using an eco-friendly shield[Table 1].Here, using the eco-friendly shield, the radiation dose reduction efficiency was $64.6 \%$ and the reduction factor was 2.8 . These results are expected to be important data for the use of eco-friendly shield in mammography.

Table 1: Comparison of Radiation Dose with and Without Eco-Friendly Shield

\begin{tabular}{lll}
\hline Scan no. & No Shield $[\mu \mathrm{Gy} \pm \mathrm{SD}]$ & eco-friendly shield $[\mu \mathrm{Gy} \pm \mathrm{SD}]$ \\
\hline 1 & $113 \pm 1.16$ & $39 \pm 0.36$ \\
2 & $113 \pm 1.06$ & $41 \pm 0.35$ \\
3 & $112 \pm 1.05$ & $40 \pm 0.33$ \\
4 & $115 \pm 1.06$ & $40 \pm 0.31$ \\
5 & $112 \pm 1.08$ & $40 \pm 0.34$ \\
Average & $113 \pm 1.06$ & $40 \pm 0.34$ \\
\% Reduction & & $64.6 \%$ \\
Reduction Factor & & 2.8 \\
\hline
\end{tabular}

\subsection{Image quality evaluation results according to use of eco-friendly shield in mammography examination}

Mammography examination is a representative site for acquiring images using low-voltage and high dose exposure conditions in simple radiography using X-ray, and since the organs exposed by $\mathrm{X}$-ray during the examination include the breast, heart, gonads, thyroid, and lens of the eye with relatively high radiation sensitivity, it is necessary to pay more attention than other areas [23].

Table 2: Evaluation Results of Image Quality According to Use of EcoFriendly Tungsten Shield during Breast Examination

\begin{tabular}{lll}
\hline Division & No Shield & eco-friendly shield \\
\hline fiber & $5.0 \pm 0.2$ & $4.5 \pm 0.0$ \\
speck & $4.5 \pm 0.0$ & $4.0 \pm 0.0$ \\
mass & $4.5 \pm 0.2$ & $4.0 \pm 0.2$ \\
total & $14.0 \pm 0.2$ & $12.5 \pm 0.1$ \\
\hline
\end{tabular}

A qualitative analysis was performed with the obtained mammograms to analyze the application of the eco-friendly tungsten shield to reduce the radiation dose. Qualitative analysis of breast tissue equivalent phantom was performed to evaluate the image quality of images with or without eco-friendly shield. Qualitative analysis consisted of evaluating the number of fibers, specks, and masses reproduced in the image by one radiology specialist and five radiologists with more than 10years experience. The standard of number of fibers was [6], and the number of specks and masses was 5 .

As a result of evaluation, when the shielding was not used, the reproducibility of fiber was $5.0 \pm 0.2$, the reproducibility of speck was 4.5 , and the reproducibility of mass was 4.5 , and by reproducing a total of 14 cases, it passed the official acceptance criteria of 10. When eco-friendly tungsten shield was used, the reproducibility of fiber was 4.5 , the reproducibility of speck was 4.0 , and the reproducibility of mass was $4.0 \pm 0.2$, totaling 12.5 , exceeding the approved acceptance criteria of 10 [Table 2].

However, the reproducibility of fiber, the reproducibility of specks, and the reproducibility of mass are different from those in the case of no shielding, which is thought to be due to the scattering due to the tungsten shield. Diagnostic radiography using X-rays should gain legitimacy because the benefit gained by the examinee is more advantageous than the risk due to the exposure, and the radiation defense optimization of the patient should be achieved[9, 23]. To achieve optimization in diagnostic radiography, much attention and will of practice is required for radiation dose reduction and radiation protection of radiation workers. For this reason, in order to minimize the radiation dose of the breast cancer examination, it is considered that the workers need to wear an eco-friendly shield.

\section{Conclusion}

To minimize the radiation dose during X-ray mammography and obtain an optimal image for diagnosis, the radiation dose distribution was measured using a glass dosimeter and an environmentally friendly tungsten shield, and the acquired image quality was evaluated.

When the eco-friendly shield was not used, the average dose was $113 \pm 1.06 \mu \mathrm{Gy}$, and when using an eco-friendly shield, the average dose was measured as $40 \pm 0.34 \mu \mathrm{Gy}$, which showed a radiation dose reduction efficiency of $64.6 \%$ and a reduction factor of 2.8 . When eco-friendly tungsten shield was used, the reproducibility of fiber was 4.5 , the reproducibility of speck was 4.0 , and the reproducibility of mass was $4.0 \pm 0.2$, totaling 12.5 , exceeding the approved acceptance criteria of 10 .

This result is expected to be used as an important basic data to decrease the radiation dose of medical care by presenting the data for predicting the radiation dose of patients and determining the method of examination in future breast cancer examination.

\section{Acknowledgment}

This research was supported by a Gimcheon University research grants in 2017.

\section{References}

[1] Eun-Hee Jang, Eun-Hoe Goo, (2017). Assessment of Detection Rate Applying the Digital Convergence Mammographic Imaging Methods (FFDM, DBT, and BMRI): Based on Breast Density. Journal of Digital Convergence, 15(5), 281-291.

[2] Ji-Hwan Cho, Hyo-Yeong Lee, In-ChulIm, (2017). Analysis of the cause by Pre Exposure Tube Voltage and Actual Exposure Tube Voltage deviation in Mammography Examination. J. Korean Soc. Radiol., Vol. 11, No. 2, 79-85.

[3] Jae-Hwan Cho, (2017). Evaluation of Usefulness of IDEAL (Iterative decomposition of water and fat with echo asymmetry and least squares estimation) Technique in 3.0T Breast MRI. Journal of Digital Contents Society, Vol. 11 No. 2, 217-224.

[4] M, Y. Kim, H. S. Kim, (2012). The evaluation of Radiation Dose by Exposure Method in Digital Magnification Mammography. The Korea Journal of Radiological Science, 35(4) 293-298.

[5] S. H. Lee, J. S. Lee, S. H. Han, (2012). A Study on Absorbed Dose in the Breast Tissue using Geant4 Simulation for Mammography. The Korea Journal of Radiological Science, 35(4), 342-352.

[6] M. H. Lee, K. R. Dong, S. J. Park, S. K. Whang, (2010). The Effect of Scattering Dose on the Thyroid during Mammography. $J$. KIEEME, Vol. 23, No. 10, 826-830.

[7] Seon-Hwa Lee, Jung-Min Kim, Dae-Cheol Kweon, (2015). Evaluation of Radiation Dose and Imaging of the QC Program in M ammography MLO View. Journal of Radiological Science and Technology, 38(3), 221-228.

[8] J. Y. Park, S. M. Baek, (2012). A Study on the Actual Conditions and Characterstics of Mammographic Units in Some Area. Journal of the Korean Scciety of Radiology, Vol6. 121-127.

[9] Chang-Gyu Kim, (2016). Exposure dose reduction during lateral spine test with water filter.Technology and Health Care, vol. 24 429-438

[10] Chang-Gyu Kim, (2016). The Development of Bismuth Shielding to Protect the Thyroid Gland in Radiations Environment. Indian Journal of Science and Technology, Vol 9(25), 1-6.

[11] Chang-Gyu Kim, Sun-Youl Seo, (2017). A Study on Reducing Exposure Dose of Radiographer Assistants during CT scan of Injury Patients without Spontaneous Breath. Jour of Adv Research in Dynamical \& Control Systems, S (10), 1-6.

[12] C. G. Kim, (2011). Measurement dose of dental panoramagraphy using a radiophotoluminescent Glass Rod Detector, The Korea Academia Industrial cooperation journal, 12(6), 2624-2628.

[13] M. Y. Jung, D. C. Kweon, S. I. Kwon, (2009). Effectiveness of Bismuth Shield to Reduce Eye Lens Radiation Dose Using the Photoluminescence Dosimetry in Computed Tomography. The Korea Society of Radiological Sceience journal, Vol. 32. No. 3, 307-312.

[14] T. J. Choi, Y. K. Oh, J. H. Kim, O. B. Kim, (2010). Development of Non-lead shielding for Radiation Protection for Medical Imaging. Medical Physics, 21st Issue 2, 232-237. 
[15] J. K. Park, I. H. Choi, H. H. Park, S. W. Yang, K. T. Kim, S. S. Kang, (2016). Design of Double Layer Shielding Structure using eco-friendly Shielding Materials. J.Korean Soc. Radiol, Vol. 10, No.8, 559-563.

[16] Y. S. Lee, J. W. Lee, Y. J. Lee, (2013). Development of the Process Mapping for the Radiation Safety Management. Journal of Radiation Protection, Vol.38, No.3, 149 - 156.

[17] Su-Jeong Yu, Sangwook Li, Sun Young Ma, Sun-YoulSeo, Young-Jae Kim, Young-Nam Kang, Ki Chang Keum, Samju Cho, (2015). Invivo Dosimetry for Mammography with and without Lead Apron Using the Glass Dosimeters. Progress in Medical Physics, Vol. 26, No. 2, 93-98.

[18] Hongmoon Jung, Jaeeun Jung, Hyejin Hyun, Doyeon Won, (2015). The Evaluation of Space Dose Distribution for Digital Mammography Equipment. J. Korean Soc. Radiol., Vol. 9, No. 1, 61-65.

[19] Chang-soo Kim, Se-Sik Kang, Jung-Hoon Kim, Jin-Soo Lee, (2014). Evaluation of Image Quality using Monte Carlo Simulation in Digital Mammography System. The Journal of the Korea Contents Association, 14(6), 247-254.

[20] Dong-Hee Hong, (2014). Evaluation of Radiolucent Considering the Compression Paddle Materials in Mammography.The Journal of the Korea Contents Association, 15(11), 307-312.

[21] ICRP, (1990). 1990 Recommendation of the international Commission on Radiological Protection, International Commission on Radiological Protection, ICRP Publication 60, Pergamon Press.

[22] C. G. Kim, (2013). Radiation dose reduction effectiveness of a male gonadal shield during 128-MDCT using Glass Detector. The Society of Digital Policy \& Management, 11(7), 237-242.

[23] C. G. Kim, (2013). Exposure dose Reduction using Pb banding of own manufacturing. The Society of Digital Policy \& Management, 11(6), 269-273. 